\title{
A Massive Sample of Radio Spectral Indices for AGN in the JVLA FIRST Survey
}

\author{
Bob Becker
}

Department of Physics, University of California Davis, USA

Email: bob@physics.ucdavis.edu

\begin{abstract}
The final 800 sq. deg of sky covered by FIRST was observed with the new, improved JVLA. The data were split between two bandpasses at 1335 and $1730 \mathrm{MHz}$ and included all four Stokes parameters, thus allowing both spectral and polarimetric results. The lower frequency bandpass data were considered part of FIRST and are available through the FIRST website (http://sundog.stsci.edu/). Here we present the higher frequency bandpass data as pertain to AGN. Foremost, we present spectral index results for the 5000 quasars with spectroscopic redshifts and the 50,000 quasars with photometric redshifts that fall in the survey area. The spectral indices are analyzed as a function of redshift and optical properties both for quasars detected above the $1 \mathrm{mJy}$ limit and, via image stacking, for quasars at flux densities down to $10 \mathrm{mJy}$.
\end{abstract}

